\title{
BIOMETRIC IDENTIFICATION BY DERMATOGLYPHICS
}

\author{
Pedro Alfonso Recobos Rodriguez \\ Division de Estudios de Posgrado e Investigacion \\ Instituto Tecnologico de Chihuahua \\ Jesus Dario Landa Silva \\ Instituto Tecnologico de Chihuahua \\ Instituto Tecnologico de Chihuahua II \\ Chihuahua, Chih., MEXICO.
}

\begin{abstract}
Biometric identification (voice, face, iris, fingerprints, etc.) of individuals takes a lot of processing due to the complexity of these patterns. In this paper we present a study by computer vision of the palmar dermatoglyphics. It is shown that the extraction and interpretation of this kind of patterns is less complicate than the patterns previously studied[1-3], they allow to obtain a particular firm for each individual which may be used for identification purposes or genetic studies[4,5]. The procedure is composed by the following steps: Image acquisition, edge detection, binary conversion, thinning operation, image reconstruction and image interpretation. Preliminary results show that this approach is a viable alternative for person identification.
\end{abstract}

\section{INTRODUCTION}

Biometric identifcation of people has been broadly studied but the most of physiologic characteristics produce patterns of considerable complexity.This work presents a computer vision study of the flexion shrinks of the palm hand creases known as dermatoglyphics[5], this marks are genetically determined as well as the fingerprints, however, they contain less information making the processing simpler.

Genetic studies had established that the palmar flexion shrinks are formed a few weeks after the conception and this patterns can only be equal in monocigotic individuals[5].

A methodology is established to measure and characterize the dermatoglyphic pattern in order to obtain a discriminating factor of identification.

It is needed to extract the flexion lines of the digitalized image, insolate the geometric structure of the dermatoglyphic pattern and represent it by a characteristic vector $\mathrm{V}=[\mathrm{W} 1, \mathrm{~W} 2, \mathrm{~W} 3, \ldots, \mathrm{Wn}][6]$.

\section{1 The Dermatoglyphics}

The dermatoglyphics studied were the flexion shrinks in the palm hand. Generally two or three of them are the principal marks in each person's hand. The digitized image is acquired with a dynamic range of 256 gray levels and a resolution of 256 X 243 (62208 pixels). This kind of images present the following characteristics that difficult its processing :

a) Low contrast between the palmar surface and its lines due to the similarity of the skin pigmentation in this area.

b) Unimodal histogram since the object occupies a very small area in the image.

c) Different brightness level for each image due to the different skin pigmentation of each individual.

Figure 1A shows an image with the conditions previously stated.

\section{PROCESS DESCRIPTION}

\section{2 . 1 Filtering}

A Sobel filter is applied so the contrast between the palmar surface and the flexion shrinks is enhanced. Fig. 1B

\section{2 . 2 Binary Image}

By means of an histogram analysis the optimal threshold is dinamically obtained for each image. An automatic selection method based on the analysis of the histogram statistical moments is applied [7].

This algorithm considers the histogram as an unidimensional density function, for each gray level the probability of occurrence is computed :

$$
P_{i}=\frac{n_{i}}{N_{T}} \quad i=0,1,2, \ldots, 255
$$

where 
$\mathrm{ni}=$ Number of image pixels with gray level $\mathrm{i}$,

$\mathrm{NT}=$ Total number of image pixels.

the first order moment for the whole histogram is obtained by :

$$
\mathrm{m} 1=\mu_{T}=\sum_{i=1}^{L}(i)\left(P_{i}\right)=\sum_{i=1}^{L} i\left(\frac{n_{i}}{N_{T}}\right)
$$

where $\mathrm{L}$ is the maximum gray level (255).

To make a binary image, the original image is divided in two classes $\mathrm{C} 0$ and $\mathrm{C} 1$ with gray intensity levels $0-\mathrm{k}$ and $\mathrm{k}+1-\mathrm{L}$ respectively. For each level $\mathrm{k}$ two cumulative moments are computed :

$$
\omega(k)=\sum_{i=1}^{k} P_{i} \quad \mu(k)=\sum_{i=1}^{k}(i)\left(P_{i}\right)
$$

the probability of the class occurrence and the mean of the class respectively.

The optimal threshold is the one that maximizes the separability between the classes which is given by:

$$
\sigma_{B}^{2}(k)=\frac{\left[\mu_{T} \omega(k)-\mu(k)\right]^{2}}{\omega(k)[1-\omega(k)]}
$$

Figure $1 \mathrm{C}$ shows the resulting image after this method is applied.

\section{2 .3 Thinning}

To obtain the geometric structure of the dermatoglyphic pattern the algorithm "Safe Point Thinning Algorithm" (SPTA)[8] is applied. This algorithm was selected due to its easy implementation and good performance compared to others[9]. Figure 1D shows the obtained image after this process.

\section{2 . 4 Pattern reconstruction.}

Once the image is thinned, the non object components are removed by a "hit-miss" transformation which is defined by:

$$
X \otimes B=\{x / B X 1 \subset X \wedge B X 2 \subset \bar{X}\}
$$

where: $\mathrm{X}=$ Original image

$\mathrm{B}=$ Structural element

$\mathrm{BX} 1=$ Belong to the object

$\mathrm{BX} 2=$ Belong to the background
The structural element specifically designed for this is shown below :

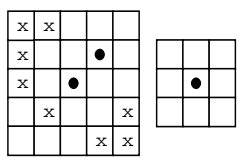

which is searched in the whole image to eliminate it. This erases single pixels and small structures ( 2 or 3 pixels) that do not belong to the palmar flexions.

To complement the pattern reconstruction, the gaps made by the acquisition and segmentation process are filled. The algorithm used is based in the coding of the obtained patterns by the use of the Freeman chain code [10]. The geometric structure obtained to this point usually contains gaps as the one shown below.

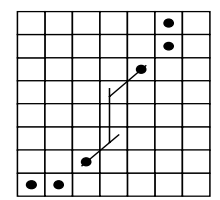

To reconstruct the dermatoglyphic pattern the next steps are followed:

a) Look for a starting arc pixel (shrink)

b) While the border exists

1)assign a Freeman code

c) Look for the nearest neighbor

d) If the separating distance is less or equal than a predetermined constant,

1) Reconstruction by the minimum

distance.

if not, look for the next shrink.

e) End

Step (c), looks for a pixel inside the area given by the coordinates:

$$
(i-1, j-8)(i-1, j+2)(i+11, j-8)(i+11, j+2)
$$

where $(i, j)$ is the coordinate of the last coded pixel of step (b). Step (d) establishes the minimum route to fill the gap, this is done by the compute of the Euclidean distance.

This algorithm has the additional advantage of erasing the remaining parasite components. Figure $1 \mathrm{E}$ shows the obtained patterns. 


\section{2 . 5 Pattern Description}

The representation methods that we used, utilize the BCC obtained during image reconstruction.

\section{5.1 Firm}

Given the "n" point sequence $(\mathrm{x} 2, \mathrm{y} 2), . .,(\mathrm{xn}, \mathrm{yn})$ of each shrink the distance from the origin to each border point is computed

$$
d_{i}=\sqrt{\left(x_{i}\right)^{2}+\left(y_{i}\right)^{2}}
$$

in this way we obtain an unidimensional function $\mathrm{f}(\mathrm{i})$ of "n" values which are different for each studied shrink.

\subsection{Fourier Descriptors}

For the same sequence of " $\mathrm{n}$ " points of each pattern, each point is represented by a complex number $\mathrm{s}(\mathrm{k})=$ $\mathrm{x}(\mathrm{k})+\mathrm{y}(\mathrm{k})$, for $\mathrm{k}=1,2, \ldots, n$. From each $\mathrm{s}(\mathrm{k})$ the DFT is computed and all the coefficients computed for the same shrink are added to form a single descriptor :

$$
S_{T}=\sum_{k=1}^{n} S(k)
$$

\subsubsection{Quantity and length of the shrinks.}

From the computed BCC the number of dermatoglyphics is counted as well as the length of each one.

\subsubsection{Statistical Moments}

For the image function $\mathrm{g}(\mathrm{x}, \mathrm{y})$ the bidimensional moments invariants are computed. These represents the shape of each dermatoglyphic pattern[11].

\section{RESULTS}

The image sequence from $\mathrm{A}$ to $\mathrm{E}$ of figure 1 shows the results in each step of the process up to the reconstruction. Images A and B of figure 2 show the result of the algorithm on another person hand. Table 1 shows the results obtained for the pattern descriptions of different persons with dermatoglyphic patterns very similar to the naked eye. Tests run on a data base of 15 images obtain a variance between the characteristic vectors of different persons of 30.48 while the variance between different vectors for the same individual is 0.4751 .

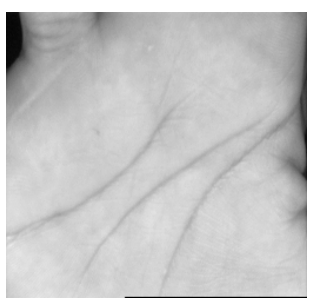

(A)

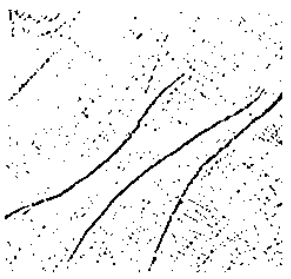

( C )

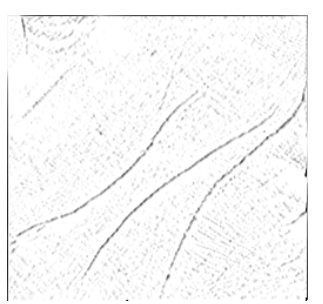

(B)

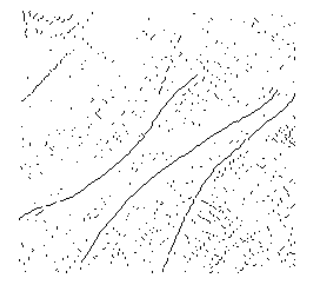

(D)

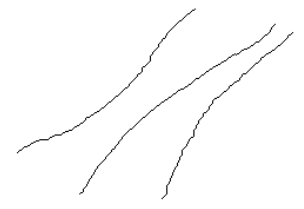

(E)

FIG. 1 (A) ORIGINAL IMAGE, (B) BORDER DETECTION, (C) BINARY IMAGE, (D) THINNING, (E) ISOLATED PATTERN

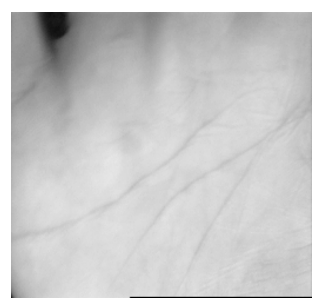

( A )

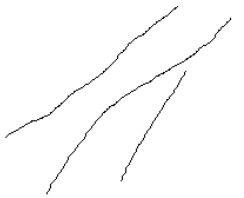

( B )
FIG. 2 (A) ORIGINAL IMAGE, (B) ISOLATED PATTERN

\section{CONCLUSIONS}

From the results we can conclude that the flexion shrinks of the palm hands are characteristics that can be used to accomplish person identification, however, it has to be noted that the image acquisition must be made in a controlled fashion. Once the dermatoglyphic pattern has been extracted, a 98\% reduction is obtained from the original image, diminishing the processing time and computer power required to perform the identification algorithm in contrast with other methods which produce too large patterns. 


\section{DIFFERENT INDIVIDUALS}

\begin{tabular}{|c|c|c|c|c|}
\hline I & II & III & IV & V \\
\hline 7.83 & 6.91 & 5.29 & 7.7 & 22.9 \\
\hline 26.5 & 20.18 & 5.32 & 0.78 & 443.5 \\
\hline 3.23 & 10.08 & 4.15 & 344.7 & 617.1 \\
\hline 2.19 & 0.30 & 0.15 & 237.8 & 273.5 \\
\hline-5.45 & 0.50 & -0.12 & 61220 & 110613 \\
\hline 11.27 & -0.12 & 0.08 & -48.5 & 5074 \\
\hline-2.06 & 0.08 & 0.00 & 29861 & 19720 \\
\hline 845.4 & 772.8 & 537.0 & 1057 & 531.9 \\
\hline 1221 & 1019 & 656.7 & 1491 & 388.6 \\
\hline 207.3 & 215.4 & 124.0 & 201.5 & 42.5 \\
\hline 251.5 & 252.4 & 119.4 & 170.8 & 48.5 \\
\hline 213.2 & 118.9 & 111.6 & 102.6 & 69.9 \\
\hline
\end{tabular}

Table I. Descriptors for different dermatoglyphic patterns corresponding to 5 different persons. (rows 1-7 invariant moments, rows 8-9 Fourier descriptors, rows 10-12 length of the shrinks). Variance between patterns is 52.079 aprox.

\section{REFERENCES}

[1] Moayer Bijan, Fu King-Sun , " A Tree System Approach for Fingerprint Pattern Recognition " , IEEE Trans. on Pattern Anal. and Machine Intel., vol. 8, pp. 376-387, 1986.

[2] Brunelli R., Falavigna D., " Person Identification using Multiple Cues " , IEEE Trans. on Pattern Anal. and Machine Intel. , vol. 17, pp. 955-955, 1995.

[3] Miller Benjamin, " Vital Signs of Identity ", IEEE Spectrum Magazine, vol 31, pp. 22-30, 1994.

[4] Cummins H., Midlo C., 1961," Fingerprints, palms and soles , An Introduction to Dermatoglyphics ", Dover Publications Inc. New York.

[5] Nora J. James, Clarke Fraser F., "Genetica Medica" , La Prensa Medica Mexicana.

[6] Adrian Low, 1991," Computer vision and Image Processing ", Mc-Graw-Hill.

[7] Noboyuky Otsu, " A Threshold Selection Method from Gray-Level Histograms", IEEE Trans. on Systems, Man and Cybernetics, vol. 1, pp. 62-66.

[8] Naccache Nabil, Shinghal Rajjan, "SPTA : A proposed Algorithm for Thinning Binary Patterns", IEEE Trans. on Systems, Man and Cybernetics, vol. 14, pp. 409-418.

[9] Lam Lousa, Lee Seong-Whan, Suen Ching, "Thinning Methodologies A Comprehensive survey", IEEE Trans. on Pattern Anal. and Machine Intel., vol. 14, pp. 869885, 1992.

[10] Gonzalez C. Rafael, Woods E. Richard, 1993, " Digital Image Processing ", Addison-Wesley.

[11] Hu Ming-Kuei, "Visual Recognition by Moments Invariants", IEEE Trans. on Information Theory", February, pp. 179-187, 1962. 